\title{
Electron-phonon interaction in quantum-dot/quantum-well semiconductor heterostructures
}

\author{
F. Comas ${ }^{1,2}$ and Nelson Studart ${ }^{2}$ \\ ${ }^{1}$ Departamento de Física Teórica, Universidad de la Habana, Vedado 10400, Havana, Cuba \\ ${ }^{2}$ Departamento de Física, Universidade Federal de São Carlos, 13565-905 São Carlos SP, Brazil
}

(Dated: November 27, 2017)

\begin{abstract}
Polar optical phonons are studied in the framework of the dielectric continuum approach for a prototypical quantum-dot/quantum-well (QD/QW) heterostructure, including the derivation of the electron-phonon interaction Hamiltonian and a discussion of the effects of this interaction on the electronic energy levels. The particular example of the CdS/HgS QD/QW is addressed and the system is modelled according to the spherical geometry, considering a core sphere of material "1" surrounded by a spherically concentric layer of material "2", while the whole structure is embedded in a host matrix assumed as an infinite dielectric medium. The strength of the electron-LO phonon coupling is discussed in details and the polaronic corrections to both ground state and excited state electron energy levels are calculated. Interesting results concerning the dependence of polaronic corrections with the QD/QW structure size are analyzed.
\end{abstract}

PACS numbers: $63.22 .+\mathrm{m}, 71.38 .-\mathrm{k}, 73.21 . \mathrm{La}$

\section{INTRODUCTION}

Quantum-dot/quantum-well (QD/QW) semiconductor heterostructures based on CdSe, ZnS, CdS, HgS, etc., have been synthesized by means of colloidal solution chemistry in the latter years. They display rather interesting size-dependent optical properties 1.2 .3 .4 .5 .6 and appear to have promising perspectives in the streaming progress of nanostructured device technology. In the case of $\mathrm{QD} / \mathrm{QW}$ heterostructures a few monolayers of material "2" (say, $\beta-\mathrm{HgS}$ ) are epitaxially grown on a core structure of material "1" (say, CdS) and, after that, the system is sometimes capped with an additional layer of material " 1 ". The whole system is embedded in a host material, which may be a silicate matrix or an organic compound. We should remark that, for $\mathrm{QD} / \mathrm{QW}$ heterostructures based on $\mathrm{CdS} / \beta-\mathrm{HgS}$, a true quantum-well is created within the QD because the bulk $\beta$-HgS energy gap is lower than the CdS gap. Highly confined carriers in the $\beta$-HgS layer were actually detected by means of different kinds of optical experiments. Besides the electronic and optical properties, the vibrational spectra are rather important in the discussion of the physical characteristics of these heterostructures. Usually the so-called dielectric continuum approach (DCA) has been applied for the theoretical determination of TO and LO bulk-like phonons, while, in the case of small-size crystal heterostructures, we must also consider the SO phonons associated to the system interfaces ${ }^{7}$. Most of the theoretical studies considered the spherical geometry ${ }^{7.8,9.10,11}$, but in some cases spheroidal QD's were investigated $12,13,14$. Theoretically more reliable approaches, as those reported in Refs. 151617 , involve rather complicated calculations. However, it must be realized that the DCA, being an essentially electrodynamical approach and relatively simple in its theoretical formalism, is still able to reveal many important physical features of the system as has been proved by all previous works on the subject.

In this paper we focus on the confined internal LO phonons, and also on the electron-LO phonon interactions for a $\mathrm{QD} / \mathrm{QW}$ heterostructure. The corresponding analysis for SO phonons was already accomplished in Ref. [18] for the same kind of model-system. By applying the methods of the DCA we derive the electric potential for confined internal LO phonons and, using this result, the electron-LO phonon interaction Hamiltonian is deduced. The electric potential allows to estimate the strength of the interaction, but the interaction effects may also be estimated by an analysis of the energy shifts of the unperturbed levels (the so-called polaronic effect). We discuss that this kind of effects is able to provide a better estimation of the electron-phonon interaction strength. Polaronic effects have been studied by different authors for systems more or less similar to the one we are discussing in the present paper. Within the adiabatic approximation, and using second order perturbation theory, the polaronic corrections were studied for nanocrystallites in Refs. 101920 , and also in Refs. 11,21,22 23 by applying other methods. It is worth to emphasize that nanostructured QD's, considering systems beyond the single QD sphere, involve more complicated vibrational properties and need to be further studied. Henceforth, in the present work we aim to show the main effects of the electron-LO phonon interactions and their influence on the energy levels of the QD/QW heterostructure by applying a relatively simple model. We show calculations involving both the ground state energy level and the excited state energy levels of the QD/QW heterostructure and comparisons are made with other previous works on the subject.

The paper is organized as follows. In Sec. II we give a brief summary of the fundamental results of the DCA and apply them to our model-system, thus deriving the internal LO phonon electric potential. In Sec. III we analyze the wave functions and energy levels in the framework of 
the effective mass approximation and give a perturbative estimation of polaronic energy shifts. Finally, Sec. IV presents a detailed discussion of the main results of the work.

\section{ELECTRIC POTENTIAL FOR THE INTERNAL LO PHONONS}

The DCA is based on the well-known Born-Huang equations, which have been extensively described in the literature ${ }^{7.8 .9 .12 .13}$ and leads to the equation:

$$
\varepsilon(\omega) \nabla^{2} \varphi=0,
$$

where $\varphi$ is the quasistatic electric potential and $\varepsilon(\omega)$ is given by the standard expression: $\varepsilon(\omega)=$ $\varepsilon_{\infty}\left(\omega^{2}-\omega_{L}^{2}\right) /\left(\omega^{2}-\omega_{T}^{2}\right)$. In the above equation the harmonic time dependence $\varphi(\boldsymbol{r}, t) \sim \exp (-i \omega t)$ is applied. We further assume the Lyddane-Sachs-Teller relation: $\omega_{L}^{2} / \omega_{T}^{2}=\varepsilon_{0} / \varepsilon_{\infty}$, while $\varepsilon_{0}\left(\varepsilon_{\infty}\right)$ is the static (high frequency) dielectric constant of the medium and $\omega_{L}, \omega_{T}$ are the bulk longitudinal and transverse phonon frequencies at the semiconductor $\Gamma$ point. The internal LO phonons satisfy Eq. (11) with $\varepsilon(\omega)=0$ and, therefore, $\omega=\omega_{L}$. Obviously, the electric potential does not satisfy the Laplace equation. Thus, the potential must be obtained as an expansion in an appropriate system of base functions fulfilling the fundamental symmetry conditions of the structure. In our case, the structure is spherically symmetric, and the spherical coordinates $(r, \theta, \phi)$ are the more convenient. Of course, the electric potential must also satisfy the usual boundary conditions: $\varphi$ should be continuous at the interface between two different media and also should satisfy the condition

$$
\varepsilon_{1}\left[\frac{\partial \varphi_{1}}{\partial n}\right]_{S}=\varepsilon_{2}\left[\frac{\partial \varphi_{2}}{\partial n}\right]_{S},
$$

i.e., the continuity of the normal component of the electric displacement $\boldsymbol{D}$. Let us also recall the rather useful relation

$$
\nabla \varphi=\frac{\varepsilon_{\infty} \omega_{L}}{\varepsilon_{\infty}-\varepsilon(\omega)} \sqrt{\frac{4 \pi \rho}{\varepsilon^{*}}} \boldsymbol{u}, \quad \frac{1}{\varepsilon^{*}}=\frac{1}{\varepsilon_{\infty}}-\frac{1}{\varepsilon_{0}},
$$

where $\rho$ is the reduced mass density addressed to the involved ion couple and $\boldsymbol{u}$ is the mechanical relative displacement vector with units of length. For the internal LO phonons Eq. (3) is applied taking $\varepsilon(\omega)=0$.

The geometry of the spherical QD/QW heterostructure is assumed in the following form: in the interval $0<r<a$ we have material "1" (a polar semiconductor like CdS), in the interval $a<r<b$ we have material "2" (a different polar semiconductor like $\mathrm{HgS}$ ), and finally for $r>b$ we shall assume an infinite dielectric medium with a fixed dielectric constant $\varepsilon_{D}$. Using spherical coordinates the electric potential is expanded as follows: for $r<a$ we apply the complete set of orthogonal functions $j_{l}(q r) Y_{l m}(\theta, \phi)$, where $j_{l}(x)$ with $l=0,1,2, \cdots$ are spherical Bessel functions and $Y_{l m}(\theta, \phi)$ are the spherical harmonics with $|m| \leq l$; for $a<r<b$ we shall apply the complete set of orthogonal functions defined by $\left[n_{l}(q a) j_{l}(q r)-j_{l}(q a) n_{l}(q r)\right] Y_{l m}(\theta, \phi)$, where $n_{l}(x)$ are spherical Bessel functions of the second kind. Let us remark that in the present model-system two different semiconductor materials are involved and two different types of phonons should be taken into account. In the central spherical core $(r<a)$ we have semiconductor " 1 ", oscillating with the frequency $\omega_{1 L}$, in the spherical layer $(a<r<b)$ we have semiconductor "2" oscillating with the frequency $\omega_{2 L}$. For the same reason we must consider two different electric potentials associated to these phonons: electric potential $\varphi^{(1)}$, due to phonons in the core sphere, and electric potential $\varphi^{(2)}$ due to phonons in the concentric spherical layer. There is not overlapping between these electric potentials: $\varphi^{(1)}$ is confined to the core sphere and must be zero for $r \geq a$, while $\varphi^{(2)}$ is confined to the spherical shell and must be zero for $r \leq a$ and $r \geq b$. The latter properties of the electric potential are a clear consequence of the boundary conditions (2) when applied to the two spherical surfaces at $r=a$ and $r=b$ respectively. On the other hand, the equation $j_{l}(q a)=0$ determines the possible values of $q$ involved in the expansion of the electric potential $\varphi^{(1)}$. For the electric potential $\varphi^{(2)}$ the possible values of $q$ present in the corresponding expansion are determined by the equation $n_{l}(q a) j_{l}(q b)=j_{l}(q a) n_{l}(q b)$.

It is useful to represent the electric potential in the following form: $\varphi_{l m}^{(i)}(r, \theta, \phi)=\Phi_{l}^{(i)}(r) Y_{l m}(\theta, \phi)$, where $\Phi_{l}^{(i)}(r)(i=1,2)$ is the radial part of the potential, which is also dependent on the various values of $q$ defining the possible phonon modes. It is then clear that for $r<a$

$$
\Phi_{l}^{(1)}(r)=A_{l m}^{(1)}(q) j_{l}(q r),
$$

and

$$
\Phi_{l}^{(2)}(r)=A_{l m}^{(2)}(q)\left[n_{l}(q a) j_{l}(q r)-j_{l}(q a) n_{l}(q r)\right],
$$

for $a<r<b$. In Eqs. (4) and (5) $A^{(i)}(q)$ are normalization constants (to be determined below) and the expansion involves summations over $q, l$, and $m$. In order to proceed with the normalization of the electric potential we should introduce the phonon annihilation and creation operators $\hat{a}_{l m}(q)$ and $\hat{a}_{l m}^{\dagger}(q)$ obeying bosonic commutation relations. Correspondingly, the classical displacement vector $\boldsymbol{u}$ is transformed into the operator $\hat{\boldsymbol{u}}$ by means of

$$
\hat{\boldsymbol{u}}^{(i)}=u_{0}^{(i)} \nabla f^{(i)}(\boldsymbol{r}) \hat{a}_{l m}(q)
$$

where

$$
\begin{aligned}
f_{q l m}^{(1)}(\boldsymbol{r}) & =j_{l}(q r) Y_{l m}(\theta, \phi), \\
f_{q l m}^{(2)}(\boldsymbol{r}) & =\left[n_{l}(q a) j_{l}(q r)-j_{l}(q a) n_{l}(q r)\right] Y_{l m}(\theta, \phi) .
\end{aligned}
$$

We should realize that the $q$ 's present in potentials of type "1" or "2" are different (determined by different transcendental equations). 
In order to determine the constants $u_{0}^{(i)}$ a well-known procedure should be applied, which has been described elsewhere $e^{8,12,13,18}$. We transform the classical kinetic energy due to the vibrations into a quantum operator, which is then taken as the LO phonon Hamiltonian $\hat{H}_{p h}$. Requiring this Hamiltonian to have its standard harmonic form, i.e., $\hat{H}_{p h}^{(i)}=\hbar \omega_{i L}\left(\hat{a}^{\dagger} \hat{a}+1 / 2\right)$ we can determine $u_{0}^{(i)}$. The calculations are straightforward and we briefly show them in the Appendix. We have found that

$$
\begin{aligned}
& u_{0}^{(1)}=\left[\frac{4 \hbar}{\rho_{1} \omega_{1 L} a x_{1}^{2} j_{l+1}^{2}\left(x_{1}\right)}\right]^{1 / 2}, \\
& u_{0}^{(2)}=\left[\frac{4 \hbar}{\rho_{2} \omega_{2 L} a x_{2}^{2} M_{l}\left(x_{2}\right)}\right]^{1 / 2},
\end{aligned}
$$

where

$$
\begin{aligned}
M_{l}(x)= & \gamma^{3}\left[j_{l}(x) n_{l+1}(\gamma x)-n_{l}(x) j_{l+1}(\gamma x)\right]^{2} \\
& -\left[j_{l}(x) n_{l+1}(x)-n_{l}(x) j_{l+1}(x)\right]^{2},
\end{aligned}
$$

and $x=q a$ are the roots of the corresponding transcendental equation: $j_{l}(x)=0$ for phonons of type " 1 " and $n_{l}(x) j_{l}(\gamma x)=j_{l}(x) n_{l}(\gamma x)$ for phonons of type " 2 ". We have also introduced the parameter $\gamma=b / a$.

By the above described method we finally obtain the normalization constants $A_{l m}^{(i)}(q)$ (notice that actually they do not depend on $m$ due to the azimuthal symmetry of our model) introduced in Eqs. (4) and (5) . In this step Eq. (3) must be applied and we obtain

$$
\begin{aligned}
& A_{l}^{(1)}\left(x_{1}\right)=\left[\frac{16 \pi \hbar \omega_{1 L}}{\varepsilon_{1}^{*} a x_{1}^{2} j_{l+1}\left(x_{1}\right)^{2}}\right]^{1 / 2}, \\
& A_{l}^{(2)}\left(x_{2}\right)=\left[\frac{16 \pi \hbar \omega_{2 L}}{\varepsilon_{2}^{*} a x_{2}^{2} M_{l}\left(x_{2}\right)^{2}}\right]^{1 / 2} .
\end{aligned}
$$

In Eqs. (12) and (13) $1 / \varepsilon_{i}^{*}=1 / \varepsilon_{i \infty}-1 / \varepsilon_{i 0}$. We have thus obtained an explicit analytic expression for the electric potential operator $\hat{\varphi}^{(i)}$ corresponding to both types of internal LO phonons involved in our model-system:

$$
\begin{aligned}
& \hat{\varphi}_{q l m}^{(1)}(r, \theta, \phi)=\frac{1}{2} A_{l}^{(1)}\left(x_{1}\right)\left[f_{q l m}^{(1)}(\boldsymbol{r}) \hat{a}_{l m}\left(x_{1}\right)+h . c .(14)\right. \\
& \hat{\varphi}_{q l m}^{(2)}(r, \theta, \phi)=\frac{1}{2} A_{l}^{(2)}\left(x_{2}\right)\left[f_{q l m}^{(2)}(\boldsymbol{r}) \hat{a}_{l m}\left(x_{2}\right)+h . c .(15)\right.
\end{aligned}
$$

where 'h.c.' stands for 'hermitian Conjugate'. Let us once again emphasize that the electric potential operator with $i=1$ is defined as the zero operator for $r>a$, while the operator with $i=2$ is the zero operator for $r<a$ and $r>b$. The electric potential operators defined in Eqs.(14) and (15) are hermitian and allow us to define the electron-LO phonon interaction Hamiltonians, which are given by

$$
\hat{H}_{e-p h}^{(i)}=-e \sum_{q l m} \hat{\varphi}_{q l m}^{(i)}(r, \theta, \phi),
$$

where $e$ is the absolute value of the electron charge. In Eq. (16) we have included a summation over all the possible eigenfunctions of this model-problem. In some expressions we are using simultaneously the notations $q$ or $x=q a$ in order to denote the roots of the corresponding transcendental equations. Whenever the notation $x_{i}$ is applied the roots of the corresponding modes for $i=1,2$ are implied. This should not lead to confusions.

\section{ELECTRON ENERGY LEVELS AND POLARONIC CORRECTIONS}

Applying the effective mass approximation for the electronic states, we model the confining potential for the electron as

$$
U(r)= \begin{cases}0 & , \text { for } 0<r<a \\ -U_{0} & , \text { for } a<r<b \\ \infty & , \text { for } r \geq b\end{cases}
$$

where $U_{0}$ is the band-offset between the conduction bands of semiconductors " 1 " and "2". We are here assuming that semiconductor "2" has a smaller energy gap and, therefore, in the shell layer $a<r<b$ the conduction electrons are actually subjected to a QW. The zero energy level was chosen at the bottom of the semiconductor "1" conduction band.

The solution of the Schrödinger equation $\hat{H} \Psi(\boldsymbol{r})=$ $E \Psi(\boldsymbol{r})$ is a straightforward problem and we are led to the wave function

$$
\Psi(r, \theta, \phi)=\psi(r) Y_{l m}(\theta, \phi)
$$

where

$\psi(r)=C \begin{cases}j_{l}\left(k_{0} r\right) & , \text { for } r \leq a \\ j_{l}\left(k_{0} a\right)\left[\frac{n_{l}(k b) j_{l}(k r)-j_{l}(k b) n_{l}(k r)}{n_{l}(k b) j_{l}(k a)-j_{l}(k b) n_{l}(k a)}\right], & \text { for } a \leq r \leq b\end{cases}$

In the above equation we have defined

$$
\begin{aligned}
k_{0} & =\sqrt{\frac{2 \mu_{1} E}{\hbar^{2}}}, \\
k & =\sqrt{\frac{2 \mu_{2}\left(U_{0}+E\right)}{\hbar^{2}}},
\end{aligned}
$$

where $\mu_{i}$ with $i=1,2$ denote the electron effective mass for each semiconductor. In this context the symbol $\mu_{0}$ stands for the free electron mass. The energy levels, denoted by $E$, are all discrete and could be positive or negative. For negative energy levels (always larger than $-U_{0}$ ) we should take $k_{0}=i \kappa$, where $\kappa$ is again given by (20) taking $|E|$ in the place of $E$. The normalization constant $C$ can be calculated requiring $\int|\Psi|^{2} d^{3} r=1$.

Notice that the wave-function described in (19) is continuous at $r=a$ and equal to zero at $r=b$. The additional boundary condition 


$$
\frac{1}{\mu_{1}}\left[\frac{\partial \psi_{1}}{\partial r}\right]_{a}=\frac{1}{\mu_{2}}\left[\frac{\partial \psi_{2}}{\partial r}\right]_{a} .
$$

leads to the discrete energy levels, given by the roots of the transcendental equation

$$
\frac{k_{0} \mu_{2}}{k \mu_{1}} \frac{j_{l}^{\prime}\left(k_{0} a\right)}{j_{l}\left(k_{0} a\right)}=\frac{n_{l}(k b) j_{l}^{\prime}(k a)-j_{l}(k b) n_{l}^{\prime}(k a)}{n_{l}(k b) j_{l}(k a)-j_{l}(k b) n_{l}(k a)} .
$$

In Eq. (23) the prime in the spherical Bessel functions denotes the first derivative with respect to the function's argument.

It is interesting to analyze the effects of the electronLO phonon interaction on the electron energy levels. For the considered semiconductors this interaction is, in general, weak and should lead to a relatively small shift towards the lower energies. Taking into account just the interaction with confined internal LO phonons we realize that, for the studied QD/QW heterostructure, two different electron-LO phonon interaction Hamiltonians are involved, as predicted by Eq. (16). For LO phonons of type "1" ("2") only the region $r<a(a<r<b)$ provides a contribution. In general, the SO phonons (analyzed in Ref. [18]) also contribute to the energy shift for all the electronic states with $l \geq 1$. For electron states with $l=0$ the SO phonons do not contribute to the energy shift. Other important issue is the degeneracy of electron states with $l \geq 1$. This degeneracy (of order $2 l+1$ ) in general could be lifted if the interaction with LO and SO phonons is taken into account. In the present work spin effects are not considered.

The general theory for the calculation of the polaronic corrections in systems similar to the one studied in this work has been described elsewhere 11.19.20.21.22 and need not be given here in detailed form. Applying the perturbative approach (and assuming that the system behaves adiabatically) we are led to the expression

$$
\Delta E_{p}=-\sum_{p^{\prime}} \sum_{i} \frac{\left|\left\langle p^{\prime}, 1\left|\hat{H}_{e-p h}^{(i)}\right| p, 0\right\rangle\right|^{2}}{E_{p^{\prime}}^{0}+\hbar \omega_{i L}-E_{p}^{0}} .
$$

In Eq.(24) we are assuming $T=0 \mathrm{~K}$, so all the LO phonons involved in the polaronic corrections are virtual. Label " $p$ " is used to describe the electronic state we are analyzing, while label " $p$ " denotes all the intermediate electronic states in the second order perturbation theory formula. $\Delta E_{p}$ gives the energy shift of level " $p$ " due to the electron-phonon coupling. The symbol $E_{p}^{0}$ corresponds to unperturbed electron energies and $|p, 1\rangle(|p, 0\rangle)$ is a ket-vector notation for the electron-one phonon (electron-zero phonon) states. In our concrete calculations the symbol " $p$ " involves three quantum numbers $(l, m, \nu)$ but the energy levels depend just on " $l, \nu$ ", where " $\nu$ " denotes the roots of the corresponding transcendental equation (Eq.(23) ) for a fixed value of $l$. If we are renormalizing the energy of an electron state with $l=0$ the calculations become easier because the corresponding level is not degenerate and the contribution from SO phonons can be ignored. States with $l \geq 1$ are obviously more complicated and require quite involved calculations. Another important issue present in Eq.(24) is the summation over all possible phonon states. However, it should be kept in mind that the possible phonon modes are, in general, bounded, i.e. the number of possible modes cannot be larger than the system volume divided by the unit cell volume. In the practical case, just the first phonon modes provide a significant contribution to the energy shift.

\section{DISCUSSION OF THE RESULTS}

Let us discuss the essential results obtained in the foregoing sections. For the numerical computations we considered that material " 1 " is CdS and material " 2 " is $\beta$ $\mathrm{HgS}$. The values of the corresponding physical parameters are given in Table I.

Table I: Semiconductor physical parameters.

\begin{tabular}{|c|c|c|c|c|c|c|}
\hline & $\varepsilon_{\infty}$ & $\varepsilon_{0}$ & $\omega_{T}\left(\mathrm{~cm}^{-1}\right)$ & $\omega_{L}\left(\mathrm{~cm}^{-1}\right)$ & $\mu / \mu_{0}$ & $E_{g}(\mathrm{eV})$ \\
\hline $\mathrm{CdS}$ & 5.5 & 9.1 & 233 & 300 & 0.2 & 2.5 \\
\hline $\mathrm{HgS}$ & 11.36 & 18.2 & 197.5 & 250 & 0.036 & 0.5 \\
\hline
\end{tabular}

We must emphasize that, within the adopted model, the properties of the dielectric host medium for $r>b$ are immaterial. Both internal LO phonons and electrons are defined for $r<b$. SO phonons, however, do depend on the properties of the host medium, but they are not considered here.

One important issue which deserves a close analysis is the strength of the electric potential associated to the two different types of internal LO phonons of our system. A first estimation can be made by a study of the radial part of the electric potentials as given in Eqs. (46) and (5). In Fig. 1 we are plotting $\Phi^{(i)}(r)$ (in units of $\Phi_{0}=\left(4 \hbar \omega_{1 L} / \pi a \varepsilon_{1}^{*}\right)^{1 / 2}$ ) for $l=0$, setting $\gamma=1.8$ and taking into account the first three roots of the corresponding transcendental equations: $j_{l}(x)=0$ or $n_{l}(x) j_{l}(\gamma x)=n_{l}(\gamma x) j_{l}(x)$ for $i=1$ or 2 . In the figure we can see the dependence of the radial part of the potential on $r$, and two regions are displayed. For the first part $(r<a)$ what we actually have is the electric potential $\Phi^{(1)}(r)$, due to LO phonons of type "1". In the second part $(a<r<b)$ the displayed potential is $\Phi^{(2)}(r)$, due to LO phonons of type "2". In Fig. 1 we have used a dotted, a dash-dotted, and a solid line in order to distinguish the three first roots of the transcendental equations in increasing order. As expected, for higher values of the corresponding root the electric potential oscillates more intensively, an effect that should led to lower values of the electron-phonon matrix elements. On the other hand, it is interesting to note that the intensity of the potential, i.e., the absolute value of 


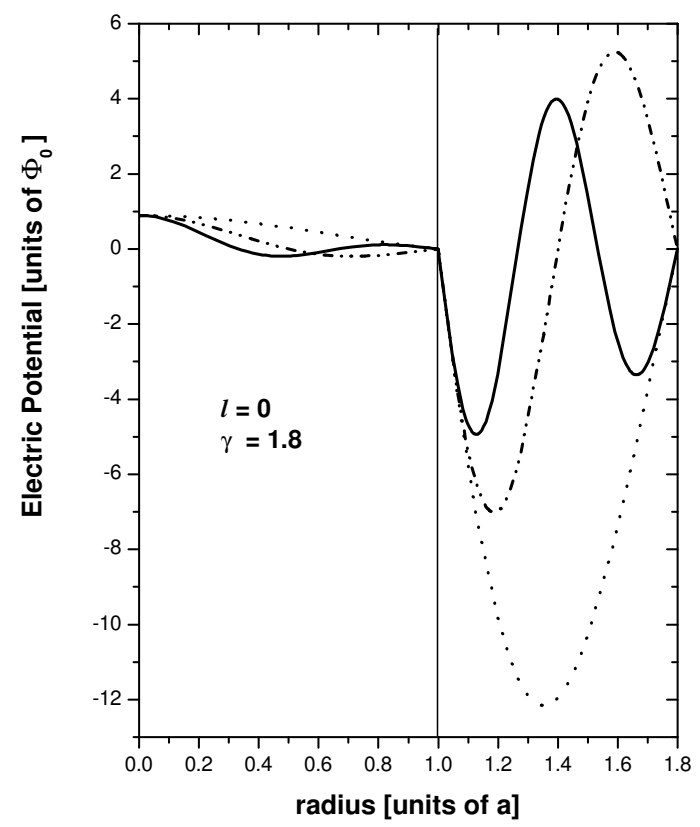

FIG. 1: Radial part of the electric potential (in units of $\Phi_{0}$ ) as a function of the radius (in units of $a$ ) for $l=0$ and $\gamma=$ 1.8. The potentials correspond to the first three LO phonon modes and are depicted by a dotted, dash-dotted and solid line respectively.

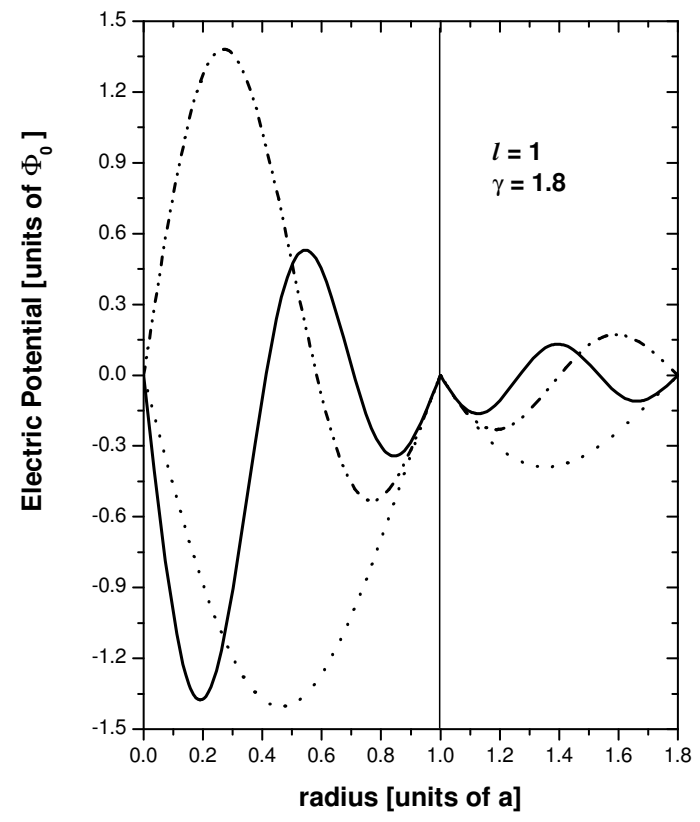

FIG. 2: Same as in Fig. 1 but now displaying the potentials for the first three LO phonon modes with $l=1$.

$\Phi^{(i)}(r)$, is lower within the core sphere $(r<a)$ in comparison with the layer region $a<r<b$. When we limit ourselves to a discussion of just the radial part of the potential, we are loosing some information concerning the angular dependence that is actually non trivial. However, the radial part provides a certain estimation of the poten-

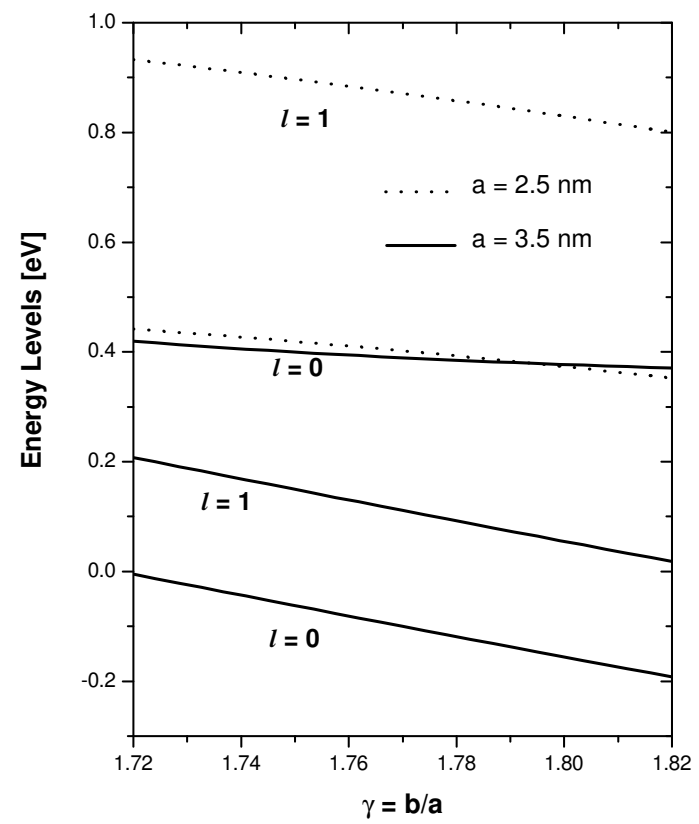

FIG. 3: Energy levels for the unperturbed CdS/HgS QD/QW heterostructure (in $\mathrm{eV}$ ) as a function of $\gamma=b / a$. For $a=2.5$ $\mathrm{nm}$ we show the first two levels (dotted lines). For $a=3.5$ $\mathrm{nm}$ (solid lines) the first three energy levels are shown. For each level the corresponding value of $l$ is explicitly indicated.

tial strength that gives an insight into the fundamental characteristics of the electron-LO phonon interaction. In Fig. 2 we analyze the radial part of the potential taking now $l=1$ and the same value for $\gamma$. Again the first three roots of the transcendental equations were considered for this value of $l$. It is evident from the figure that, in contrast with the results shown in Fig. 1, in the present case the potential intensity is higher inside the core region. From the latter results it could be inferred that the electron should feel a stronger phonon interaction in the region $r<a$ for $l=1$, while for $l=0$ the electron-phonon interaction should be stronger in the region $a<r<b$. However, this kind of analysis could be certainly misleading. In order to properly understand the strength of the electron-phonon interaction we must also take into account the electron wave functions. For instance, if for $l=0$ we get a larger probability amplitude of finding the electron inside the core sphere, then this region could provide the larger contribution to the electron-phonon interaction. For that reason the strength of the interaction is better revealed in such quantities as relaxation rates, polaronic corrections, etc., where the matrix elements of the electron-phonon interaction Hamiltonian must be calculated.

In Fig. 3 we are showing (solid lines) the first three energy levels of the $\mathrm{QD} / \mathrm{QW}$ heterostructure as a function of $\gamma=b / a$. These results were obtained from Eq.(23) without the inclusion of the electron-phonon (polaronic) corrections. For the core sphere radius we have taken $a=3.5 \mathrm{~nm}$, while the band-offset between the conduc- 
tion bands of CdS and $\beta-\mathrm{HgS}$ was estimated as $U_{0}=1.34$ $\mathrm{eV}$ following Ref. 24]. For this value of $a$ the first (ground state) energy level is localized inside the QW region $(E<0$ for our choice of the zero energy level at the bottom of the CdS conduction band). The first excited energy level (with $l=1$ ) is already out of the QW with $E>0$ and the second excited level (with $l=0$ ) can also be seen in the figure. The values of $\gamma$ were chosen in a relatively narrow interval $1.72<\gamma<1.82$. According to reported experimental results.1.2.3.4, $\gamma$ is bound to be not very large. On the other hand, for lower values of $\gamma$ there are not possible levels for $E<0$. For this energy level the wave function in the region $r<a$ is expressed through hyperbolic functions, while for other energy levels wavefunctions are given by oscillating trigonometric functions. The latter fact provides important changes in the calculation of matrix elements and leads to differences in polaronic energy shifts (to be discussed below). In Fig. 3, using dotted lines, we show the first two energy levels for a core sphere radius with $a=2.5 \mathrm{~nm}$. It is clear from the figure that, in this latter case, there are not energy levels with $E<0$ in the analyzed interval for $\gamma$. In Fig. 3 we are not showing the third energy level for $a=2.5 \mathrm{~nm}$ with $l=0$. This level has the energy $E \approx 1.30 \mathrm{eV}$ when $\gamma=1.72$ decreasing down to $E \approx 1.08$ $\mathrm{eV}$ for $\gamma=1.8$.

As discussed in Section III, the electron-phonon interaction leads to an energy shift of the electronic levels towards the lower energies, the so-called "polaronic effect". Polaronic effects in the electron, hole, and exciton energy levels for QD structures have been studied by different authors 10.11 .19 .20 .21 .22 . In most of the published papers the single spherical QD was considered, while the applied theoretical formalism is in some cases perturbative and in other cases the variational one. For a system like the one studied in the current paper, it is plausible to apply a perturbative approach as was briefly described in Section III. Polaronic corrections may be discussed considering both the ground state energy level and the excited states levels, and usually involve tedious lengthy calculations. In order to make our calculations we applied Eq.24) where the labels $p$ and $p^{\prime}$ were adapted to our system. The electron-LO phonon interaction was evaluated according to Eq. (16) considering both types of LO phonons present in the $\mathrm{QD} / \mathrm{QW}$ heterostructure and including summations over an appropriate amount of phonon modes. On the other hand, electron wavefunctions, properly normalized, were taken according to Eq.(19) and the discrete energy levels were calculated by solving the transcendental equation (23).

In Fig. 4 we present the absolute value of the polaronic energy shift corresponding to the case with $a=2.5$ $\mathrm{nm}$. We show the corrections to the ground state energy level (the first dotted curve in Fig. 3) for a relatively wide range of $\gamma$ values. These energy shifts, given here in meV, should be subtracted from the corresponding energies reported in Fig. 3. The energy shifts are decreasing for growing values of $\gamma$, while the calculated

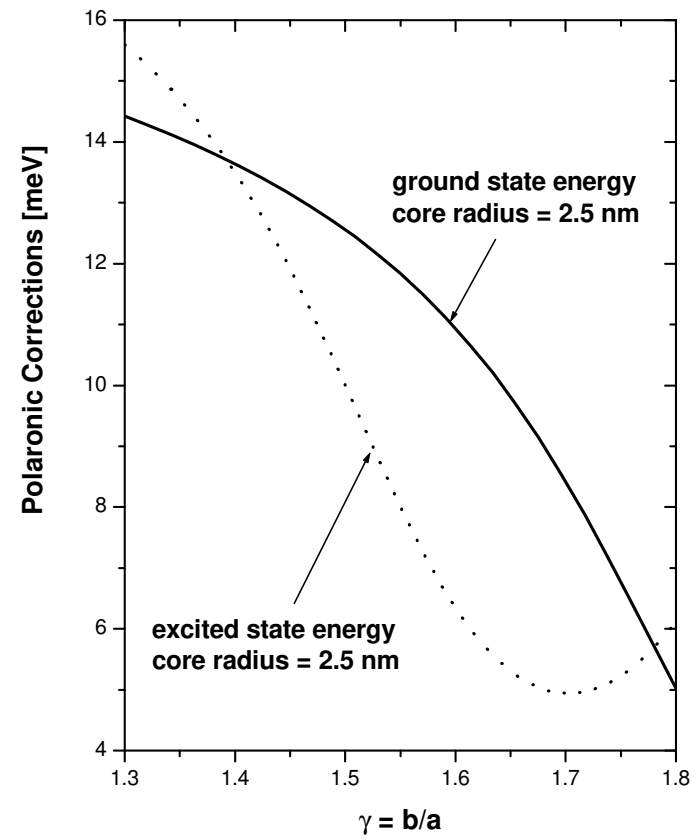

FIG. 4: Absolute value of the energy shifts (in meV) as a function of $\gamma=b / a$ for the polaronic corrections to the ground state (solid line) and excited state (dotted line) energy levels considering a QD/QW heterostructure with core radius $a=$ $2.5 \mathrm{~nm}$; These energy shifts should be subtracted from the corresponding energy levels presented in Fig. 3.

values are relatively low as could be expected for a system having a weak electron-LO phonon coupling. The obtained results disagree with those reported in Ref. 21] for a similar situation in which the opposite trend for the polaronic corrections was obtained. In Fig. 4 we also show the polaronic corrections to the excited energy level (dotted curve). This energy level, as mentioned above, has $l=0$ and was not shown in Fig. 3. The curve, giving the dependence with $\gamma$, shows a decreasing behavior in its first part but, after reaching a minimum approximately at $\gamma=1.7$, shows an increasing behavior for the last part of the considered interval. This peculiar kind of dependence with the thickness of the HgS layer seems to characterize the polaronic corrections for the excited levels and was in a certain way commented in Ref. [22]. It must also be remarked that, for most of the $\gamma$ interval, the corrections to the excited level are lower, than those to the ground state energy level.

In Fig. 5 we present the same dependencies as in Fig. 4, but taking now $a=3.5$. As easily seen in the figure the polaronic corrections to the excited state (considering again the state with $l=0$, i.e., the third solid line in Fig. 3) are now considerably higher than the corrections related to the ground state energy level. Actually, the polaronic energy shifts corresponding to the ground state energy level are quite low and difficult to detect in the experimental measurements. The latter results, in sharp contrast with those described in Fig. 4, can be easily understood from the physical point of view. The 


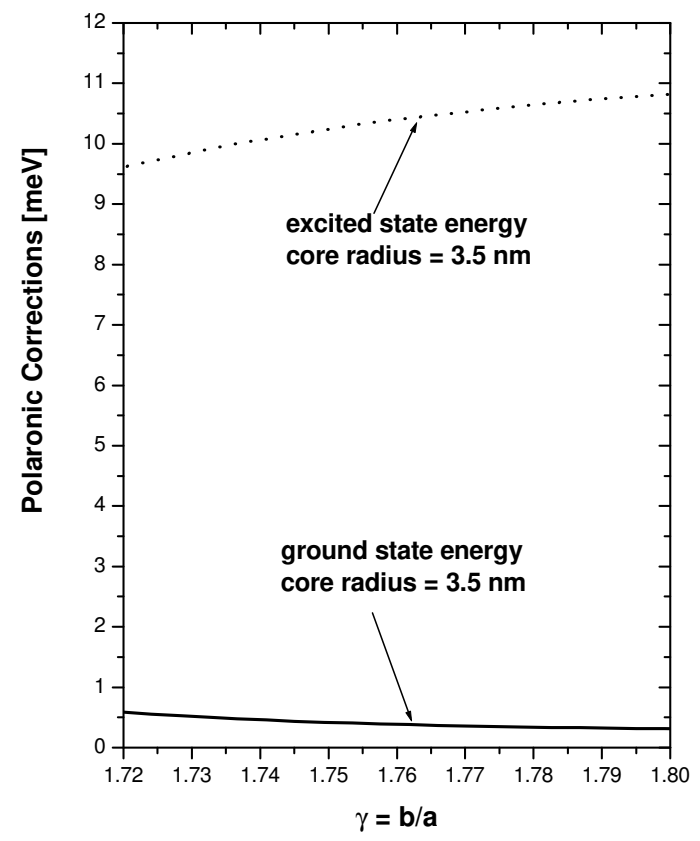

FIG. 5: Same as in Fig. 4 but taking $a=3.5 \mathrm{~nm}$. In both cases the results correspond to the $\mathrm{CdS} / \mathrm{HgS} \mathrm{QD} / \mathrm{QW}$ heterostructure.

ground state wave function (for $a=3.5 \mathrm{~nm}$ ) in the region $r<a$ is given by hyperbolic functions (in the framework of the used model-system), thus leading to very low probability amplitudes if compared with states having $E>0$. We must also notice that in Fig. 4 we are considering a narrower interval for $\gamma$ in order to ensure the presence of the ground state energy with $E<0$.

As general conclusions to the present work, we can emphasize that polaronic corrections are actually interesting in the study of the electronic energy spectra of QD/QW heterostructures. They can be detected in the appropriate optical experiments, differences between the ground state and excited states polaronic corrections may be studied and should provide good estimations of the electron-LO phonon coupling in this kind of heterostructures. Concerning degenerate energy levels (not studied in the current work) the electron-LO phonon interaction could introduce detectable energy splittings between the degenerate levels, a subject that requires further analysis.

\section{Appendix A. Normalization of the electric poten- tial}

When we substitute the hermitized operator version of $\boldsymbol{u}^{2}$, i.e., $\boldsymbol{u}^{2} \rightarrow\left(\hat{\boldsymbol{u}}^{\dagger} \cdot \hat{\boldsymbol{u}}+\hat{\boldsymbol{u}} \cdot \hat{\boldsymbol{u}}^{\dagger}\right) / 2$, in the classical expression for the kinetic energy due to the ion vibrations, we are directly led to:

$$
\hat{H}_{p h}^{(i)}=\frac{1}{2} \rho_{i} \omega_{i L}^{2} u_{i 0}^{2} \int_{V_{i}} \nabla f^{(i) *} \cdot \nabla f^{(i)} d^{3} r\left(\hat{a}^{\dagger} \hat{a}+1 / 2\right),
$$

where Eq.(6) was applied and $f^{(i)}(\boldsymbol{r})$ are the two functions given in Eqs. (7) and (8). In the above expressions, for the sake of brevity, we are avoiding some subscripts. The volumes $V_{i}$ are: for $i=1$ the sphere of radius $a$ and for $i=2$ the spherical shell defined by $a<r<b$. Integrating by parts the integral in Eq. (A1), we first obtain a surface integral that is always zero because of the boundary conditions fulfilled by the mentioned functions (notice that these functions are zero over the spherical surfaces defining the boundaries of volumes $V_{1}$ and $V_{2}$ respectively). Taking into account that

$$
\nabla^{2} f^{(i)}=-q^{2} f^{(i)}
$$

the integral can be casted as

$$
\int_{V_{i}} \nabla f^{(i) *} \cdot \nabla f^{(i)} d^{3} r=q^{2} \int_{V_{i}} f^{(i) *} f^{(i)} d^{3} r .
$$

The latter integral contains an integration over the angular part of the functions that is easily evaluated in the form: $\int Y_{l m}^{*} Y_{l m} d \Omega=1$. There remain two integrals over the radial parts that are different for $i=1,2$ and given by

$$
\begin{gathered}
\int_{0}^{a} j_{l}^{2}(q r) r^{2} d r=\frac{a^{3}}{2} j_{l+1}^{2}(q a) \\
\int_{a}^{b}\left[n_{l}(q a) j_{l}(q r)-j_{l}(q a) n_{l}(q r)\right]^{2} r^{2} d r=\frac{a^{3}}{2} M_{l}(q a)
\end{gathered}
$$

where $M_{l}(x)$ was given in Eq. (11). For the integral (A44) the condition $j_{l}(q a)=0$ is assumed, while for the integral (A5) the corresponding condition is $n_{l}(q a) j_{l}(q b)=$ $j_{l}(q a) n_{l}(q b)$. The latter results, together with the analysis made in Section II, lead to the values of $u_{0}^{(i)}$ (reported in Eqs. (9) and (10)). Using now the relation

$$
A_{l}^{(i)}(q)=\sqrt{4 \pi \rho_{i} \omega_{i L} / \varepsilon_{i}^{*}} u_{0}^{(i)}
$$

we finally obtain the normalization constants for the electric potential reported in Eqs. (12) and (13).

\section{Acknowledgments}

The work is partially supported by grants from the FAPESP (Fundação de Amparo à Pesquisa de São Paulo) and Conselho Nacional de Desenvolvimento Científico e Tecnológico (CNPq). F.C. is grateful to Departamento de Física, Universidade Federal de São Carlos, for hospitality. 
1 A. Mews, A. Eychmüller, M. Giersig, D. Schooss, and J. Weller, J. Phys. Chem. 98, 934 (1994).

2 D. Schooss, A. Mews, A. Eychmüler, and H. Weller, Phys. Rev B 49, 17072 (1994)

3 A. Mews, A.V. Kadavanich, U. Banin, and A.P. Alivisatos, Phys. Rev. B 53, R13242 (1996).

4 F. Koberling, A. Mews, and T. Basché, Phys. Rev. B 60, 1921 (1999).

5 H.E. Porteanu, E. Lifshitz, M. Pflughoefft, A. Eychmüller, and H. Weller, Phys. Stat. Sol. (b) 226, 219 (2001)

6 M. Shim, C. Wang, and P. Guyot-Sionnest, J. Phys. Chem B 105, 2369 (2001)

7 R. Ruppin and R. Englman, Rep. Prog. Phys. 33, 149 (1970) and references therein.

8 M.C. Klein, H. Hache, D. Ricard, and C. Flytzanis, Phys. Rev. B 42, 11123 (1990).

9 S. Nomura, and T. Kobayashi, Phys. Rev. B 45, 1305 (1992).

10 D.V. Melnikov, and W .B. Fowler, Phys. Rev. B 64, 245320 (2001).

11 M.T. Kach, V. Holovatsky, O. Voitsekhivska, M. Mykhalyova, and R. Fartushynsky, Phys. Stat. Sol. (b) 225, 331 (2001).

12 F. Comas, C. Trallero-Giner, N. Studart, and G.E. Marques, Phys. Rev.B 65, 073303 (2002).
13 F. Comas, C. Trallero-Giner, N. Studart, and G.E. Marques, J. Phys.: Condens. Matter 14, 6469 (2002).

14 D.A. Romanov, V.V. Mitin, and M.A. Stroscio, Phys. Rev. B 66, $115321(2002)$

15 E. Roca, C. Trallero-Giner, and M. Cardona, Phys. Rev. B 49, 13704 (1994).

16 F. Comas and C. Trallero-Giner, Physica B 192, 394 (1993).

17 C. Trallero-Giner and F. Comas, Phil. Magazine B, 70, 583 (1994).

18 F. Comas and C. Trallero-Giner, Phys. Rev. B 67, 115301 (2003).

19 J.S. Pan and H.B. Pan, Phys. Stat. Sol. (b) 148, 129 (1988).

20 S.N. Klimin, E.P. Pokatilov, and V.M. Fomin, Phys. Stat. Sol. (b) 184, 373 (1994).

21 N.V. Tkach, Phys. Sol. State 39, 995 (1997).

${ }^{22}$ K. Oshiro, K. Akai, and M. Matsuura, Phys. Rev. B 58, 7986 (1998).

23 Ph. Lelong and S.H. Lin, Appl. Phys. Lett. 81, 1002 (2002).

24 Su-Huai Wei and A. Zunger, Appl. Phys. Lett. 72, 2011 (1998). 